\title{
Using concave optimization methods for inexact quadratic programming problems with an application to waste management
}

\author{
Sumati Mahajan', S.K. Gupta², Izhar Ahmad ${ }^{3 *}$ (D) and S. Al-Homidan³
}

\section{"Correspondence:}

drizhar@kfupm.edu.sa

${ }^{3}$ Department of Mathematics \&

Statistics, King Fahd University of

Petroleum \& Minerals, Dhahran,

31261, Kingdom of Saudi Arabia

Full list of author information is

available at the end of the article

\begin{abstract}
Quadratic programming is potentially capable of strategic decision making in real world problems. However, practical problems rarely conform to crisp parameters, and hence the prospects of these problems with inexact parameters are inevitably higher. The existing studies regarding public welfare schemes/ organizations reveal that their objectives end up as minimization of cost functions and are governed by linear or concave quadratic programming problems. The present study proposes a method that can be applied to concave type quadratic objective function subject to linear constraints with inexact parameters. A comparison is also drawn with existing methods to establish its simplicity and efficiency. Further, a numerical example is illustrated, and finally, a waste management problem is formulated and solved using the proposed method.
\end{abstract}

MSC: $90 C 70 ; 90 C 20 ; 90 C 26 ; 90 C 90$

Keywords: Concave optimization; Waste management; Inexact parameters; Quadratic programming problem

\section{Introduction}

Waste management (WM) problem is paramount, in particular, both to the public and environment. Human rate of consumption and consequently production of various wastes has far outrun nature's capacity to rejuvenate. Improper handling of the problem can cause considerable damage and can even trigger a catastrophic situation. WM affects our daily activities and is of prime concern to the environmentalists, administrators, and industry. The WM issue has drawn huge attention from researchers with focus on different aspects of this problem. Mathematicians, in particular, are trying to fit a suitable programming model with imprecise parameters, which can in general be applied to WM. Primarily, linear programming (LP) model with interval parameters happens to be the first choice due to its simplicity. These models have been used to find solution of many real life problems viz. WM, ground water allocation, and flood control.

There have been many studies related to WM on various directions. Chang and Lu [1] worked on creating WM planning alternatives in view of fuzzy environmental resources.

(c) The Author(s) 2021. This article is licensed under a Creative Commons Attribution 4.0 International License, which permits use sharing, adaptation, distribution and reproduction in any medium or format, as long as you give appropriate credit to the original author(s) and the source, provide a link to the Creative Commons licence, and indicate if changes were made. The images or other third party material in this article are included in the article's Creative Commons licence, unless indicated otherwise in a credit line to the material. If material is not included in the article's Creative Commons licence and your intended use is not permitted by statutory regulation or exceeds the permitted use, you will need to obtain permission directly from the copyright holder. To view a copy of this licence, visit http://creativecommons.org/licenses/by/4.0/. 
Further, Sakai and Hiraoka [2] investigated WM solution by focussing on recycling of incinerator residue. Thereafter, Cai et al. [3] proposed a model with a combination of interval, fuzzy, and stochastic approach for WM. Next, Fan et al. [4] introduced a solution method for fuzzy LP to reduce computational requirements in the WM problem. Sun et al. [5] worked on the WM problem using a constrained quadratic model and provided an effective tool for reflecting system cost variations. Further, Tan et al. [6] identified optimal plans in fuzzy environment using a two-step solution method. On a different note, Lu et al. [7] and He et al. [8] focussed on controlling gas emission in WM using dynamic optimization and mixed integer programming approach, respectively. Thereafter, Astrup et al. [9] investigated the incinerator performance linked to emissions and residues. Li and Chen [10] suggested fuzzy stochastic interval parameters in LP model for WM. Next, Fan and Huang [11] proposed a two-step method for WM to avoid loss of decision related information, while Li and Huang [12] demonstrated a robust quadratic programming model to handle long term WM planning and decisions. Later, Ionescu et al. [13] stressed on an integrated WM model using pretreatment and revenue generating processes. Rada et al. [14] proposed an integrated solution for WM by maintaining a balance between energy and environment. Next, Xu et al. [15] presented a model to deal with possibilistic or probabilistic uncertainties and tackle complexities derived from capacity-expansion issues.

In reference to the various methodologies proposed in the literature, Sugimoto et al. [16] introduced a parallel relaxation method for quadratic programming problems. Later, Van Thorai [17] presented a finite branch and bound algorithm as an optimization technique for a quadratic programming problem. Further, Gao et al. [18] proposed a rectangle branch and reduce approach for faster convergence of nonconvex quadratic programming algorithm. Next, Liu and Wang [19] proposed a numerical solution method for interval quadratic programming. Fan et al. [20] illustrated an effective robust method for interval LP in uncertain environmental decision making. Thereafter, Fan et al. [21] described generalized fuzzy method for uncertainties in the form of fuzzy sets and developed a method using $\alpha$-cut and membership functions.

On a different note, Atalay and Apaydin [22] suggested a method based on gamma distribution for stochastic programming model while a method for nonlinearly optimization using quadratic programming was proposed by Zhu et al. [23]. Next, an algorithm for generalized vector quasi-complementarity problems with an application to traffic network problems was proposed by Van Hung et al. [24]. Recently, Van Hung et al. [25] discussed convergence of solution sets for fuzzy optimization problems, and then a new class of generalized multiobjective games with fuzzy mappings was proposed by Van Hung et al. [26]. Further, Van Hung and Keller [27] improvised the traffic network problem in [24] to a traffic network under uncertainty by establishing convergence of fuzzy vector quasioptimization problems.

In the present day scenario of a continuously changing and competitive market, the decision making has to be handled with utmost care when crisp parameters fail to provide a satisfactory solution. The volatile data in practical problems can be conveniently accommodated using interval parameters in comparison to all other types of imprecise parameters. Using interval parameters in an LP model, Li et al. [28] studied flood control planning. Further, Li and Huang [29] introduced a stochastic quadratic programming method to handle uncertainties and nonlinearities in water management. Later, Fan et al. [30] explained a generalized fuzzy LP model for air quality management. Further, Jin et al. [31] 
investigated allocation of water for irrigation purposes. Miao et al. [32] worked on an interval fuzzy programming method for water resource planning. An inexact water quality management model supporting economic and environmental management was proposed by Zhang et al. [33]. Thereafter, Huang et al. [34] studied controlling of noise in urban localities with the help of interval data. Though the LP models with interval parameters take care of the uncertainty part, governing all costs by a constant function may not tackle every practical situation.

As an application to WM under imprecise environment with linearly decreasing costs, Chen and Huang [35] initiated the application of quadratic programming problem using inexact parameters. The solution of resulting concave quadratic programming problem was found by applying derivative algorithm. The WM problem was further investigated by Kong et al. [36] and solved using duality approach in an effort to give an alternate method. However, duality results are applicable under certain conditions without which the results are misleading and do not comply with the characteristics of duality theorem. The present article not only provides an alternate strategy to tackle the WM problem, but also highlights and rectifies the discrepancy in Kong et al. [36].

The proposed algorithm is based on a two-level interval programming procedure which ultimately yields an interval solution of the given problem. The remaining part of the article is organized as follows. Section 2 reviews some basic definitions. Section 3 develops a methodology to solve inexact concave quadratic programming problems. Section 4 illustrates an example to display the steps involved in the proposed methodology. Section 5 deals with the application of inexact quadratic programming in the WM problem using the proposed approach. Next, a counterexample is constructed to describe the discrepancy in Kong et al. [36]. Further, computational efficiency of the proposed algorithm and some limitations of the inexact quadratic programming model are also discussed. Finally, conclusions with some future directions are presented in Sect. 6.

\section{Basic definitions}

Throughout the paper, $\mathbb{R}$ denotes the set of real numbers. Some of the definitions related to interval parameters are given below.

Definition 2.1 ([36]) An interval number $A^{ \pm}$is defined as an interval with known upper and lower bounds, but unknown distribution information and is defined as follows:

$$
A^{ \pm}=\left[A^{-}, A^{+}\right]=\left[a \in \mathbb{R} \mid A^{-} \leq a \leq A^{+}\right]
$$

where $A^{-}$and $A^{+}$are the lower and upper bounds of $A^{ \pm}$, respectively.

Arithmetic operations on intervals

Suppose that $A^{ \pm}$and $B^{ \pm}$are two intervals, then for $k_{1}, k_{2} \in \mathbb{R}$,

$$
k_{1}\left[A^{-}, A^{+}\right]+k_{2}\left[B^{-}, B^{+}\right]= \begin{cases}{\left[k_{1} A^{-}+k_{1} B^{-}, k_{1} A^{+}+k_{2} B^{+}\right]} & \text {if } k_{1} \geq 0, k_{2} \geq 0, \\ {\left[k_{1} A^{-}+k_{2} B^{+}, k_{1} A^{+}+k_{2} B^{-}\right]} & \text {if } k_{1} \geq 0, k_{2} \leq 0, \\ {\left[k_{1} A^{+}+k_{2} B^{-}, k_{1} A^{-}+k_{2} B^{+}\right]} & \text {if } k_{1} \leq 0, k_{2} \geq 0, \\ {\left[k_{1} A^{+}+k_{2} A^{+}, k_{1} A^{-}+k_{2} B^{-}\right]} & \text {if } k_{1} \leq 0, k_{2} \leq 0 .\end{cases}
$$


Definition 2.2 ([36]) The ordering on two intervals $A^{ \pm}$and $B^{ \pm}$is defined as follows:

$$
\begin{aligned}
& A^{ \pm} \leq B^{ \pm} \quad \text { iff } A^{-} \leq B^{-} \text {and } A^{+} \leq B^{+}, \\
& A^{ \pm}<B^{ \pm} \quad \text { iff } A^{-}<B^{-} \text {and } A^{+}<B^{+} .
\end{aligned}
$$

\section{The inexact quadratic programming problem}

To capture real world uncertainties, usual precise parameters do not serve the purpose. Among various methods to handle imprecise data, use of inexact or interval parameters is one of the simplest ways to handle realistic problems. An inexact quadratic programming problem can be formulated in the following manner:

$$
\begin{aligned}
& \text { Minimize } Z=\sum_{j=1}^{n}\left(C_{j}^{ \pm} x_{j}+D_{j}^{ \pm} x_{j}^{2}\right) \\
& \text { subject to } \sum_{j=1}^{n} A_{i j}^{ \pm} x_{j} \leq B_{i}^{ \pm}, \quad i=1,2, \ldots, m, x_{j} \geq 0, j=1,2, \ldots, n,
\end{aligned}
$$

where, for all $i$ and $j, C_{j}^{ \pm}=\left[C_{j}^{-}, C_{j}^{+}\right], D_{j}^{ \pm}=\left[D_{j}^{-}, D_{j}^{+}\right], A_{i j}^{ \pm}=\left[A_{i j}^{-}, A_{i j}^{+}\right]$, and $B_{i}^{ \pm}=\left[B_{i}^{-}, B_{i}^{+}\right]$. Further, it is assumed that $D_{j}^{+} \leq 0$ for all $j$.

As $Z$ fluctuates due to inexact parameters in an objective function as well as in constraints, it lies between two values $Z^{-}$and $Z^{+}$(say), that is, $Z=\left[Z^{-}, Z^{+}\right]$. Now, to obtain these lower and the upper bounds of (IQP), the problem is further divided into the following two sub-problems:

$$
\begin{aligned}
Z^{-}= & \min _{S}\left(\min _{x}\left(\sum_{j=1}^{n}\left(C_{j} x_{j}+D_{j} x_{j}^{2}\right)\right)\right. \\
& \text { subject to } \left.\sum_{j=1}^{n} A_{i j} x_{j} \leq B_{i}, x_{j} \geq 0, i=1,2, \ldots, m, j=1,2, \ldots, n\right)
\end{aligned}
$$

and

$$
\begin{aligned}
& Z^{+}=\max _{S}\left(\min _{x}\left(\sum_{j=1}^{n}\left(C_{j} x_{j}+D_{j} x_{j}^{2}\right)\right)\right. \\
& \left.\quad \text { subject to } \sum_{j=1}^{n} A_{i j} x_{j} \leq B_{i}, x_{j} \geq 0, i=1,2, \ldots, m, j=1,2, \ldots, n\right),
\end{aligned}
$$

where $S=\left\{\left(C_{j}, D_{j}, A_{i j}, B_{i}\right) \mid C_{j} \in\left[C_{j}^{-}, C_{j}^{+}\right], D_{j} \in\left[D_{j}^{-}, D_{j}^{+}\right], A_{i j} \in\left[A_{i j}^{-}, A_{i j}^{+}\right], B_{i} \in\left[B_{i}^{-}, B_{i}^{+}\right], \forall i\right.$ and $j$.

Now, both the problems above involve bi-level optimization criteria (max-min type for $Z^{+}$and min-min type for $Z^{-}$) with varying feasible region. Hence, to compute the bounds, it is necessary to convert these problems into single level optimization models with fixed feasible region. Before discussing the equivalent problems to evaluate these bounds, first we shall compute the smallest and largest feasible region of the problem (IQP). Consider 
the following extreme cases of the feasible region:

$$
\begin{aligned}
& S_{1}=\left\{x \in \mathbb{R}^{n} \mid \sum_{j=1}^{n} A_{i j}^{+} x_{j} \leq B_{i}^{-}, x_{j} \geq 0, \forall i, j\right\}, \\
& S_{2}=\left\{x \in \mathbb{R}^{n} \mid \sum_{j=1}^{n} A_{i j}^{-} x_{j} \leq B_{i}^{+}, x_{j} \geq 0, \forall i, j\right\}, \\
& S_{3}=\left\{x \in \mathbb{R}^{n} \mid \sum_{j=1}^{n} A_{i j}^{+} x_{j} \leq B_{i}^{+}, x_{j} \geq 0, \forall i, j\right\}, \\
& S_{4}=\left\{x \in \mathbb{R}^{n} \mid \sum_{j=1}^{n} A_{i j}^{-} x_{j} \leq B_{i}^{-}, x_{j} \geq 0, \forall i, j\right\} .
\end{aligned}
$$

Let $x \in S_{1}$ and as $A_{i j}^{-} \leq A_{i j}^{+}, B_{i}^{-} \leq B_{i}^{+}, \forall i$ and $j$, therefore

$$
\sum_{j=1}^{n} A_{i j}^{-} x_{j} \leq \sum_{j=1}^{n} A_{i j}^{+} x_{j} \leq B_{i}^{-} \leq B_{i}^{+}, \quad \forall i .
$$

Hence, by the above inequality $x \in S_{2}$. Similarly, $x \in S_{3}$ implies $x \in S_{2}$ and $x \in S_{4}$ yields $x \in S_{2}$. This implies $S_{1} \cup S_{2} \cup S_{3} \cup S_{4}=S_{2}$. Thus, the largest feasible region of (IQP) is $S_{2}$. Further, $x \in S_{1}$ yields $x \in S_{2}, x \in S_{3}$, and $x \in S_{4}$. That is, $S_{1} \subseteq S_{2}, S_{1} \subseteq S_{3}, S_{1} \subseteq S_{4}$ and hence $S_{1} \cap S_{2} \cap S_{3} \cap S_{4}=S_{1}$. This proves that the smallest feasible region of (IQP) is $S_{1}$.

Next, we shall discuss the problems to evaluate $Z^{-}$and $Z^{+}$.

\subsection{Model to compute $Z^{-}$}

Observe the following facts about the problem (LIQP):

1. It has the same nature of optimization (min-min type).

2. $C_{j}^{-} \leq C_{j}, D_{j}^{-} \leq D_{j}, x_{j} \geq 0$ for all $j$ and hence

$$
\sum_{j=1}^{n}\left(C_{j}^{-} x_{j}+D_{j}^{-} x_{j}^{2}\right) \leq \sum_{j=1}^{n}\left(C_{j} x_{j}+D_{j} x_{j}^{2}\right) .
$$

3. Among all the feasible regions, the largest feasible region $S_{2}$ defined by the constraints of the problem (LIQP) yields the best minimum value of the objective function,

it finally reduces to

$$
\begin{aligned}
& Z^{-}=\min _{x}\left(\sum_{j=1}^{n}\left(C_{j}^{-} x_{j}+D_{j}^{-} x_{j}^{2}\right)\right) \\
& \quad \text { subject to } \sum_{j=1}^{n} A_{i j}^{-} x_{j} \leq B_{i}^{+}, \quad i=1,2, \ldots, m, x_{j} \geq 0, j=1,2, \ldots, n .
\end{aligned}
$$

\subsection{Model to compute $Z^{+}$}

To solve (UIQP), which involves different nature of optimization viz. max-min type, we proceed as follows: 
As $C_{j} \leq C_{j}^{+}, D_{j} \leq D_{j}^{+}$, and $x_{j} \geq 0$ for all $j$, therefore

$$
\sum_{j=1}^{n}\left(C_{j} x_{j}+D_{j} x_{j}^{2}\right) \leq \sum_{j=1}^{n}\left(C_{j}^{+} x_{j}+D_{j}^{+} x_{j}^{2}\right) .
$$

This further implies

$$
\min _{x}\left(\sum_{j=1}^{n}\left(C_{j} x_{j}+D_{j} x_{j}^{2}\right)\right) \leq \min _{x}\left(\sum_{j=1}^{n}\left(C_{j}^{+} x_{j}+D_{j}^{+} x_{j}^{2}\right)\right) .
$$

Hence

$$
\max _{S}\left(\min _{x}\left(\sum_{j=1}^{n}\left(C_{j} x_{j}+D_{j} x_{j}^{2}\right)\right)\right)=\max _{S^{\prime}}\left(\min _{x}\left(\sum_{j=1}^{n}\left(C_{j}^{+} x_{j}+D_{j}^{+} x_{j}^{2}\right)\right)\right),
$$

where $S^{\prime}=\left\{\left(A_{i j}, B_{i}\right) \mid A_{i j} \in\left[A_{i j}^{-}, A_{i j}^{+}\right], B_{i} \in\left[B_{i}^{-}, B_{i}^{+}\right], \forall i\right.$ and $\left.j\right\}$.

Therefore, the problem (UIQP) is equivalent to

$$
\begin{aligned}
& Z^{+}=\max _{S^{\prime}}\left(\min _{x}\left(\sum_{j=1}^{n}\left(C_{j}^{+} x_{j}+D_{j}^{+} x_{j}^{2}\right)\right)\right) \\
& \quad \text { subject to } \sum_{j=1}^{n} A_{i j} x_{j} \leq B_{i}, \quad x_{j} \geq 0, i=1,2, \ldots, m, j=1,2, \ldots, n .
\end{aligned}
$$

Further, from the varying feasible regions, following best/ worst value criterion, we get

$$
\min _{S_{2}, x}\left(\sum_{j=1}^{n}\left(C_{j}^{+} x_{j}+D_{j}^{+} x_{j}^{2}\right)\right) \leq \min _{S^{\prime}, x}\left(\sum_{j=1}^{n}\left(C_{j}^{+} x_{j}+D_{j}^{+} x_{j}^{2}\right)\right) \leq \min _{S_{1}, x}\left(\sum_{j=1}^{n}\left(C_{j}^{+} x_{j}+D_{j}^{+} x_{j}^{2}\right)\right)
$$

This yields

$$
\max _{S^{\prime}}\left(\min _{x}\left(\sum_{j=1}^{n}\left(C_{j}^{+} x_{j}+D_{j}^{+} x_{j}^{2}\right)\right)\right)=\min _{S_{1}, x}\left(\sum_{j=1}^{n}\left(C_{j}^{+} x_{j}+D_{j}^{+} x_{j}^{2}\right)\right) .
$$

That is, out of all the possible, feasible regions corresponding to $S^{\prime}$, the best value of $Z^{+}$ will be obtained over the smallest region $S_{1}$ since it gives the worst value of

$$
\min _{x}\left(\sum_{j=1}^{n}\left(C_{j}^{+} x_{j}+D_{j}^{+} x_{j}^{2}\right)\right)
$$

So, the problem of finding $Z^{+}$is finally reduced to

$$
\begin{aligned}
& Z^{+}=\min _{x}\left(\sum_{j=1}^{n}\left(C_{j}^{+} x_{j}+D_{j}^{+} x_{j}^{2}\right)\right) \\
& \quad \text { subject to } \sum_{j=1}^{n} A_{i j}^{+} x_{j} \leq B_{i}^{-}, \quad i=1,2, \ldots, m, x_{j} \geq 0, j=1,2, \ldots, n .
\end{aligned}
$$


Figure 1 Flow chart of proposed algorithm

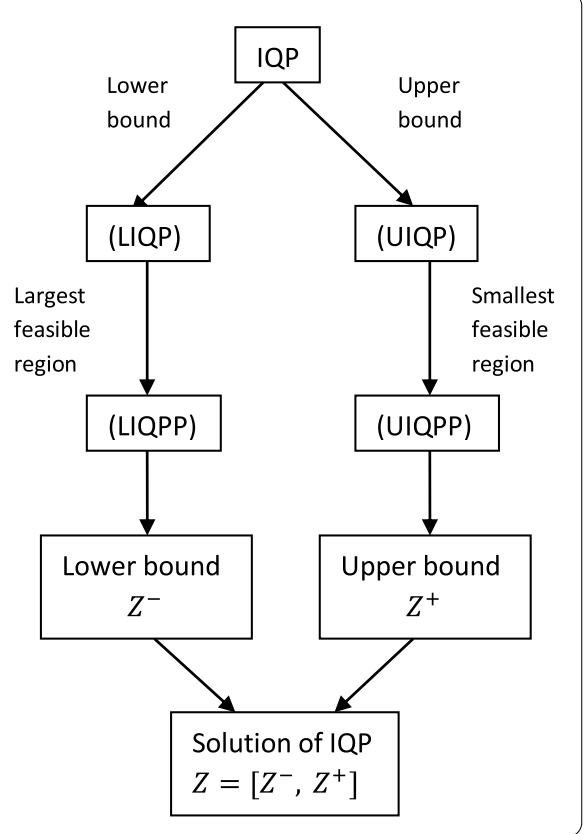

\section{Remark 1}

1. It is to be noted that, for all $j, D_{j}^{-} \leq 0$ and $D_{j}^{+} \leq 0$, which yield that the Hessian matrices of the problems (LIQPP) and (UIQPP) are negative semi-definite, and hence the problems are concave optimization models.

2. Due to the concave nature of the objective functions of (LIQPP) and (UIQPP), duality theory is also not applicable as shown in Kong et al. [36].

The following theorem guarantees the global optimal solution if the objective function (minimization type) is concave.

Theorem 1 ([37]) Consider the following problem:

$$
\begin{aligned}
& \text { Minimize } Z(x) \\
& \text { over } S=\left\{x: G_{i}(x) \leq 0, i=1,2, \ldots, m\right\},
\end{aligned}
$$

where $Z$ is a concave function defined on $\mathbb{R}^{n}, G_{i},(i=1,2, \ldots, m)$ are convex functions defined on $\mathbb{R}^{n}$ whose gradients are continuous, and $S$ is compact such that a point in the strict interior of the feasible region exists. Then there exists an extreme point $\hat{x}$ of the convex compact set $S$ which globally minimizes the problem $(\mathrm{P})$.

Flow chart representation of the proposed algorithm can be viewed in Fig. 1.

\section{Numerical example}

$$
\begin{gathered}
\text { Minimize } f=[2,5] x_{1}+[3,4] x_{2}-[3,6] x_{1}^{2}-[4,5] x_{2}^{2} \\
\text { subject to }[2,3] x_{1}+[3,4] x_{2} \leq[10,12], \\
{[2,3] x_{1}+[1,2] x_{2} \geq[4,6],}
\end{gathered}
$$




$$
x_{1}, x_{2} \geq 0 .
$$

Solution: Using LIQPP and UIQPP models to find lower and upper bound, respectively, the two subproblems are:

$$
\begin{aligned}
& \text { Lower bound: } f^{-}=\text {Minimize } 2 x_{1}+3 x_{2}-6 x_{1}^{2}-5 x_{2}^{2} \\
& \text { subject to } 2 x_{1}+3 x_{2} \leq 12, \\
& 3 x_{1}+2 x_{2} \geq 4, \\
& x_{1}, x_{2} \geq 0, \\
& \text { Upper bound: } f^{+}=\text {Minimize } 5 x_{1}+4 x_{2}-3 x_{1}^{2}-4 x_{2}^{2} \\
& \text { subject to } 3 x_{1}+4 x_{2} \leq 10, \\
& 2 x_{1}+x_{2} \geq 6, \\
& x_{1}, x_{2} \geq 0 .
\end{aligned}
$$

Noting that the objective functions of the above problems are negative semi-definite, the model happens to be concave optimization. Using MAPLE-12, we get $f^{-}=-204$ (at $\left.x_{1}=6, x_{2}=0\right)$ and $f^{+}=-16.67$ (at $x_{1}=3.33, x_{2}=0$ ). Hence, $f=[-204,-16.67]$.

\section{Application to waste management}

The proposed method is applied to a WM study (Fig. 2) on three localities and two WM facilities viz. the landfill and the incinerator. Total time span is fifteen years, which is split into three time spans of five years each. Due to impreciseness in all of collection and transportation costs, landfill and incinerator operating costs, residue disposal cost, and earnings from generated energy sales, an interval quadratic programming model is most appropriate. The capacity of the landfill is approximately $3.75 \times 10^{6}$ to $4.00 \times 10^{6}$ tonnes and the incinerator capacity is 500 to 650 tonnes per day. It is observed that the incinerator leaves residues of nearly $30 \%$ of the incoming waste. The problem is to allocate appropriate waste flows from the localities to the suitable facility while minimizing the aggregate cost.

Taking into account the transportation costs, running costs of facilities, and earnings from energy generated out of the incinerator as some of the factors while determining the problem, the IQP problem to be investigated is formulated as follows:

Minimize $f=$ the sum of

(1) Collection and transportation cost: $\sum_{i=1}^{2} \sum_{j=1}^{3} \sum_{k=1}^{3} L_{k} T R_{i j k}^{ \pm} x_{i j k}$,

(2) Running cost of the landfill/ incinerator: $\sum_{i=1}^{2} \sum_{j=1}^{3} \sum_{k=1}^{3} L_{k} O P_{i k}^{ \pm} x_{i j k}$,

(3) Cost of disposal of incinerator residue in the landfill: $\sum_{j=1}^{3} \sum_{k=1}^{3} L_{k} F E\left(F T_{k}^{ \pm}+O P_{2 k}^{ \pm}\right) x_{2 j k}$

(4) Earnings from sale of energy: $-\sum_{j=1}^{3} \sum_{k=1}^{3} L_{k} R E_{k}^{ \pm} x_{2 j k}$ subject to

(I) Landfill capacity constraint: $\sum_{j=1}^{3} \sum_{k=1}^{3} L_{k}\left(x_{1 j k}+F E x_{2 j k}\right) \leq T L^{ \pm}$,

(II) Incinerator capacity constraint: $\sum_{j=1}^{3} x_{2 j k} \leq T E_{k}^{ \pm}, \forall k$,

(III) Waste disposal demand constraint: $\sum_{i=1}^{2} x_{i j k} \geq W G_{j k}^{ \pm}, \forall j, k$,

(IV) Nonnegativity constraint: $x_{i j k} \geq 0, \forall i, j, k$. 


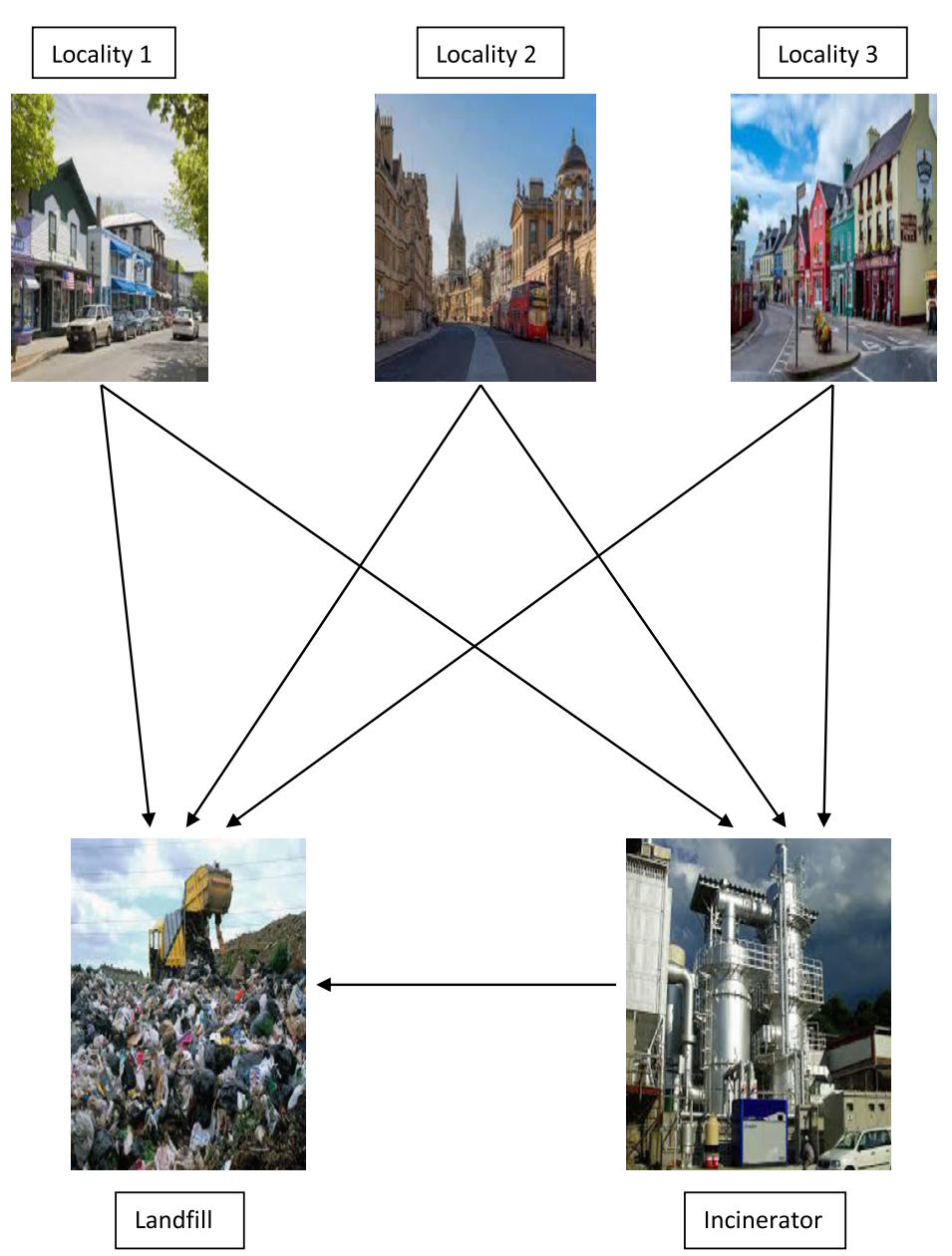

Figure 2 Waste management system

Table 1 Waste generation data (in tonnes/day)

\begin{tabular}{llll}
\hline & Time span $(k=1)$ & Time span $(k=2)$ & Time span $(k=3)$ \\
\hline$W G_{1 k}^{ \pm}$ & {$[250,350]$} & {$[300,400]$} & {$[350,440]$} \\
$W G_{2 k}^{ \pm}$ & {$[150,250]$} & {$[185,265]$} & {$[215,285]$} \\
$W G_{3 k}^{ \pm}$ & {$[250,350]$} & {$[250,350]$} & {$[300,400]$} \\
\hline
\end{tabular}

Table 2 Facility running cost (in dollars)

\begin{tabular}{llll}
\hline & Time span $(k=1)$ & Time span $(k=2)$ & Time span $(k=3)$ \\
\hline$O P_{1 k}^{ \pm}$ & {$[35,37.5]$} & {$[50,53]$} & {$[64,65]$} \\
$O P_{2 k}^{ \pm}$ & {$[62.5,63]$} & {$[75,77]$} & {$[83.5,87.5]$} \\
\hline
\end{tabular}

where:

$f$ is the aggregate cost of waste disposal $(\$)$,

$i=1,2$ stands for the facility; ( 1 for the landfill and 2 for the incinerator),

$j=1,2,3$ stands for the locality,

$k=1,2,3$ stands for the time span,

$L_{k}$ is length of the time span $k(=1825$ days for all $k)$, 
Table 3 Waste transportation cost (in dollars)

\begin{tabular}{llll}
\hline & Time span $(k=1)$ & Time span $(k=2)$ & Time span $(k=3)$ \\
\hline$T R_{11 k}^{ \pm}$ & {$[-0.0161 X+15.08$,} & {$[-0.0181 X+16.02$,} & {$[-0.0195 X+17.65$,} \\
$T R_{12 k}^{ \pm}$ & $-0.0121 X+19.50]$ & $-0.0139 X+21.30]$ & $-0.0146 X+23.45]$ \\
$T R_{13 k}^{ \pm}$ & {$[-0.0142 X+12.63$,} & {$[-0.0146 X+13.92$,} & {$[-0.0169 X+15.31$,} \\
$T R_{21 k}^{ \pm}$ & $-0.0105 X+16.89]$ & $-0.0116 X+18.58]$ & $-0.0127 X+20.35]$ \\
$T R_{22 k}^{ \pm}$ & {$[-0.0170 X+15.30$,} & {$[-0.0188 X+16.84$,} & {$[-0.0209 X+18.45$,} \\
$T R_{23 k}^{ \pm}$ & $-0.0130 X+20.50]$ & $-0.0142 X+22.54]$ & $-0.0158 X+24.70]$ \\
& {$[-0.0130 X+11.55$,} & {$[-0.0144 X+12.75$,} & {$[-0.0155 X+14.01$,} \\
& $-0.0098 X+15.40]$ & $-0.0109 X+16.97]$ & $-0.0118 X+18.66]$ \\
& {$[-0.0136 X+12.17$,} & {$[-0.0149 X+13.40$,} & {$[-0.0164 X+14.75$,} \\
& $-0.0103 X+16.15]$ & $-0.0113 X+17.75]$ & $-0.0124 X+19.55]$ \\
& {$[-0.0120 X+10.05$,} & {$[-0.0130 X+11.65$,} & {$[-0.0143 X+12.83$,} \\
& $-0.0090 X+14.02]$ & $-0.0098 X+15.52]$ & $-0.0108 X+17.06]$ \\
\hline
\end{tabular}

Table 4 Transportation cost from the incinerator to the landfill (in dollars)

\begin{tabular}{llll}
\hline & Time span $(k=1)$ & Time span $(k=2)$ & Time span $(k=3)$ \\
\hline$F T_{k}^{ \pm}$ & {$[-0.0065 X+5.70$,} & {$[-0.0070 X+6.27$,} & {$[-0.0076 X+6.90$,} \\
& $-0.0047 X+7.65]$ & $-0.0055 X+8.38]$ & $-0.0058 X+9.25]$ \\
\hline
\end{tabular}

Table 5 Earnings (dollars) and capacity of the incinerator (in tonnes/day)

\begin{tabular}{llll}
\hline & Time span $(k=1)$ & Time span $(k=2)$ & Time span $(k=3)$ \\
\hline$R E_{k}^{ \pm}$ & {$[15,20]$} & {$[15,20]$} & {$[15,20]$} \\
$T E_{k}^{ \pm}$ & {$[500,650]$} & {$[500,650]$} & {$[500,650]$} \\
\hline
\end{tabular}

$x_{i j k}$ is the waste transferred to facility $i$ from locality $j$ during time span $k$ (tonnes/day), $T R_{i j k}^{ \pm}$is collection and transportation cost from locality $j$ to facility $i$ during time span $k$ (\$/tonne), $O P_{i k}^{ \pm}$is running cost of facility $i$ during time span $k$ (\$/tonne),

$F E$ is residue flow rate from the incinerator to the landfill (30\%),

$F T_{k}^{ \pm}$is transportation cost for residue from the incinerator to the landfill during time span $k$ (\$/tonne),

$R E_{k}^{ \pm}$is earnings from the incinerator in time span $k$ (\$/tonne),

$T L^{ \pm}$is capacity of the landfill $\left(3.75 \times 10^{6}-4.00 \times 10^{6}\right.$ tonnes $)$,

$T E_{k}^{ \pm}$is capacity of the incinerator in time span $k$ (tonnes/day),

$W G_{j k}^{ \pm}$is waste generation rate in locality $j$ during time span $k$ (tonnes/day).

The waste collection and transportation cost are governed by market trend of decreasing linear functions, and in particular for locality 1, represented by Figs. 3-6. Similarly, transportation cost for residue from the incinerator to the landfill is also observed following decreasing linear function trend.

Using data in the given model from Tables $1-5$, the inexact quadratic programming problem becomes:

$$
\begin{aligned}
\operatorname{minimize} f= & 1825\left([50.08,57] x_{111}+[74.51,84.595] x_{211}+[47.63,54.39] x_{121}\right. \\
& +[75.13,85.345] x_{221}+[50.30,58] x_{131}+[73.01,83.215] x_{231} \\
& +[66.02,74.3] x_{112}+[92.13,104.584] x_{212}+[63.92,71.58] x_{122} \\
& +[92.78,105.364] x_{222}+[66.84,75.54] x_{132}+[91.03,103.13] x_{232}
\end{aligned}
$$




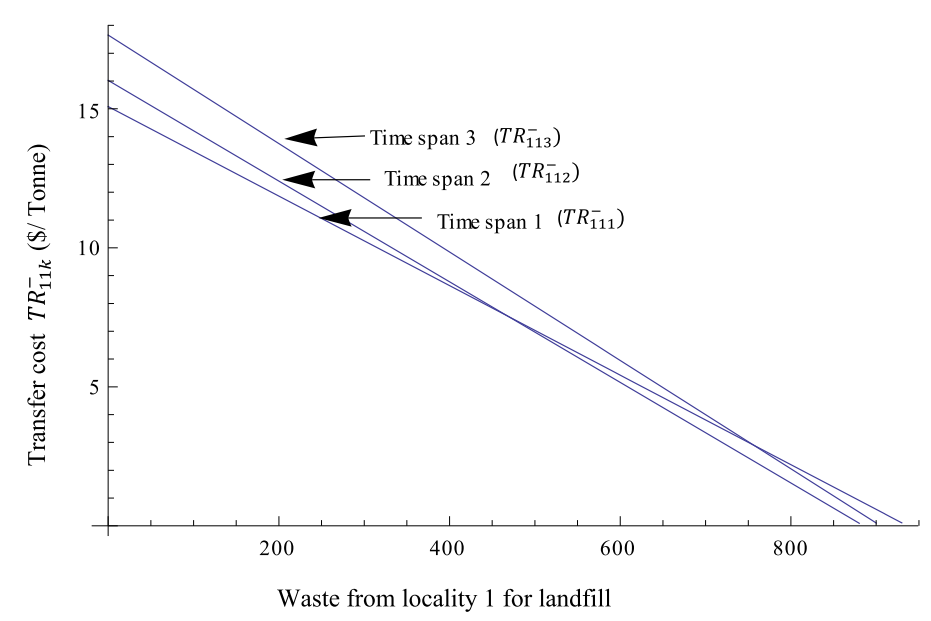

Figure 3 Lower bound of waste collection and transfer cost from locality 1 to the landfill

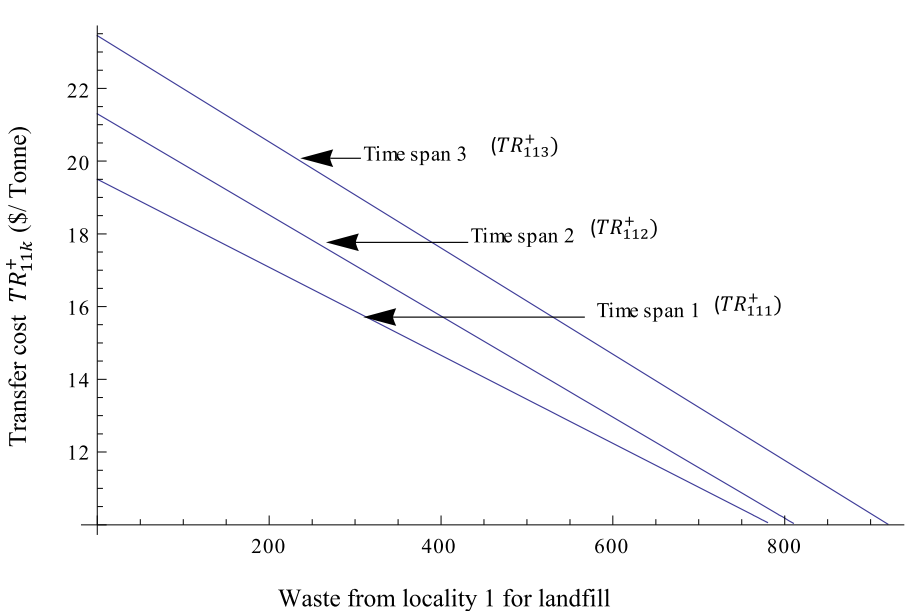

Figure 4 Upper bound of waste collection and transfer cost from locality 1 to the landfill

$$
\begin{aligned}
& +[81.65,88.45] x_{113}+[104.63,120.19] x_{213}+[79.31,85.35] x_{123} \\
& +[105.37,121.075] x_{223}+[82.45,89.7] x_{133}+[103.45,118.585] x_{233} \\
& +[-0.0161,-0.0121] x_{111}^{2}+[-0.01495,-0.01121] x_{211}^{2} \\
& +[-0.0142,-0.0105] x_{121}^{2}+[-0.01555,-0.01441] x_{221}^{2} \\
& +[-0.0170,-0.0130] x_{131}^{2}+[-0.01395,-0.01041] x_{231}^{2} \\
& +[-0.0181,-0.0139] x_{112}^{2}+[-0.0165,-0.01255] x_{212}^{2} \\
& +[-0.0146,-0.0116] x_{122}^{2}+[-0.017,-0.01295] x_{222}^{2} \\
& +[-0.0188,-0.0142] x_{132}^{2}+[-0.0151,-0.01145] x_{232}^{2} \\
& +[-0.0195,-0.0146] x_{113}^{2}+[-0.01778,-0.01354] x_{213}^{2} \\
& +[-0.0169,-0.0127] x_{123}^{2}+[-0.01868,-0.01414] x_{223}^{2}
\end{aligned}
$$




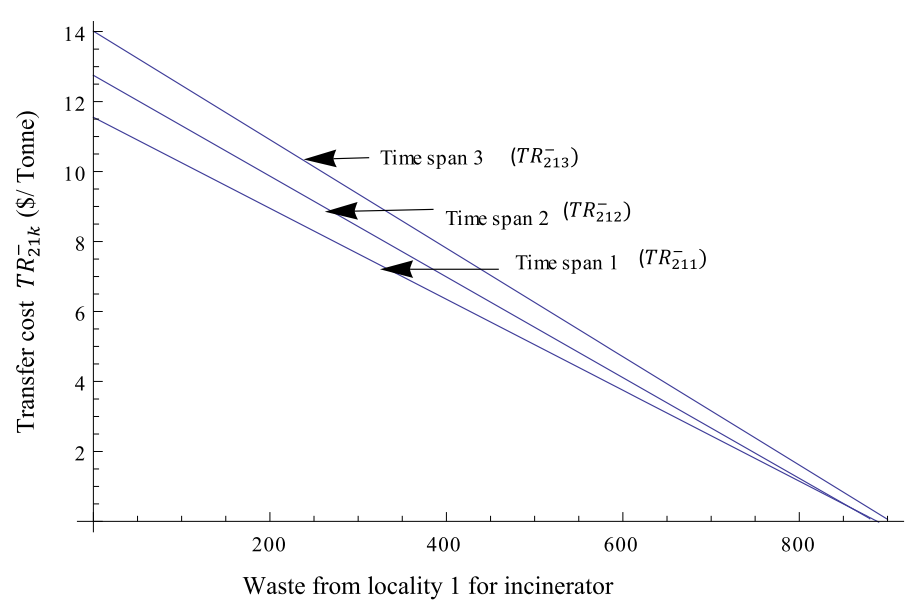

Figure $\mathbf{5}$ Lower bound of waste collection and transfer cost from locality 1 to the incinerator

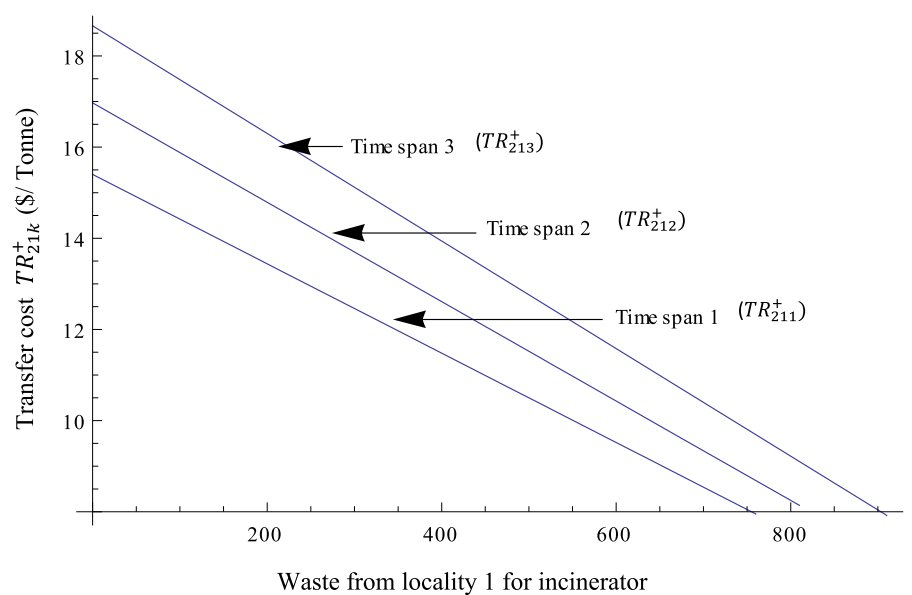

Figure 6 Upper bound of waste collection and transfer cost from locality 1 to incinerator

$$
\left.+[-0.0209,-0.0158] x_{133}^{2}+[-0.01658,-0.01254] x_{233}^{2}\right)
$$

subject to

$$
\begin{aligned}
& 1825\left(x_{111}+x_{121}+x_{131}+x_{112}+x_{122}+x_{132}+x_{113}+x_{123}+x_{133}\right. \\
& \left.+0.3\left(x_{211}+x_{221}+x_{231}+x_{212}+x_{222}+x_{232}+x_{213}+x_{223}+x_{233}\right)\right) \\
& \leq[3.75,4.00] \times 10^{6} \text {, } \\
& x_{211}+x_{221}+x_{231} \leq[500,650] \text {, } \\
& x_{212}+x_{222}+x_{232} \leq[500,650] \text {, } \\
& x_{213}+x_{223}+x_{233} \leq[500,650] \text {, } \\
& x_{111}+x_{211} \geq[250,350] \text {, } \\
& x_{121}+x_{221} \geq[150,250] \text {, }
\end{aligned}
$$




$$
\begin{aligned}
& x_{131}+x_{231} \geq[250,350], \\
& x_{112}+x_{212} \geq[300,400], \\
& x_{122}+x_{222} \geq[185,265], \\
& x_{132}+x_{232} \geq[250,350], \\
& x_{113}+x_{213} \geq[350,440], \\
& x_{123}+x_{223} \geq[215,285], \\
& x_{133}+x_{233} \geq[300,400], \\
& x_{i j k} \geq 0 \quad \forall i, j, k .
\end{aligned}
$$

Now, using the model (LIQPP), we have

$$
\begin{aligned}
f^{-}= & \min _{x}\left(1 8 2 5 \left(50.08 x_{111}+74.51 x_{211}+47.63 x_{121}+75.13 x_{221}+50.30 x_{131}\right.\right. \\
& +73.01 x_{231}+66.02 x_{112}+92.131 x_{212}+63.92 x_{122}+92.781 x_{222}+66.84 x_{132} \\
& +91.031 x_{232}+81.65 x_{113}+104.63 x_{213}+79.31 x_{123}+105.37 x_{223}+82.45 x_{133} \\
& +103.45 x_{233}-0.0161 x_{111}^{2}-0.01495 x_{211}^{2}-0.0142 x_{121}^{2}-0.01555 x_{221}^{2} \\
& -0.0170 x_{131}^{2}-0.01395 x_{231}^{2}-0.0181 x_{112}^{2}-0.0165 x_{212}^{2}-0.0146 x_{122}^{2}-0.017 x_{222}^{2} \\
& -0.0188 x_{132}^{2}-0.0151 x_{232}^{2}-0.0195 x_{113}^{2}-0.01778 x_{213}^{2}-0.0169 x_{123}^{2}-0.01868 x_{223}^{2} \\
& \left.\left.-0.0209 x_{133}^{2}-0.01658 x_{233}^{2}\right)\right)
\end{aligned}
$$

subject to

$$
\begin{aligned}
& 1825\left(x_{111}+x_{121}+x_{131}+x_{112}+x_{122}+x_{132}+x_{113}+x_{123}+x_{133}\right. \\
& \left.\quad+0.3\left(x_{211}+x_{221}+x_{231}+x_{212}+x_{222}+x_{232}+x_{213}+x_{223}+x_{233}\right)\right) \\
& \leq 4.00 \times 10^{6}, \\
& x_{211}+x_{221}+x_{231} \leq 650, \\
& x_{212}+x_{222}+x_{232} \leq 650, \\
& x_{213}+x_{223}+x_{233} \leq 650, \\
& x_{111}+x_{211} \geq 250 \\
& x_{121}+x_{221} \geq 150 \\
& x_{131}+x_{231} \geq 250 \\
& x_{112}+x_{212} \geq 300 \\
& x_{122}+x_{222} \geq 185 \\
& x_{132}+x_{232} \geq 250 \\
& x_{113}+x_{213} \geq 350 \\
& x_{123}+x_{223} \geq 215 \\
& \quad
\end{aligned}
$$


Table 6 Optimal values of variables for lower bound $f^{-}$

\begin{tabular}{|c|c|c|c|}
\hline Time span $(k)$ & Facility type (i) & Locality (j) & Optimum waste allocation (tonnes) \\
\hline \multirow[t]{6}{*}{1} & 1 & 1 & $x_{111}=250$ \\
\hline & & 2 & $x_{121}=150$ \\
\hline & & 3 & $x_{131}=250$ \\
\hline & 2 & 1 & $x_{211}=0$ \\
\hline & & 2 & $x_{221}=0$ \\
\hline & & 3 & $x_{231}=0$ \\
\hline \multirow[t]{6}{*}{2} & 1 & 1 & $x_{112}=300$ \\
\hline & & 2 & $x_{122}=102$ \\
\hline & & 3 & $x_{132}=250$ \\
\hline & 2 & 1 & $x_{212}=0$ \\
\hline & & 2 & $x_{222}=83$ \\
\hline & & 3 & $x_{232}=0$ \\
\hline \multirow[t]{7}{*}{3} & 1 & 1 & $x_{113}=350$ \\
\hline & & 2 & $x_{123}=215$ \\
\hline & & 3 & $x_{133}=300$ \\
\hline & 2 & 1 & $x_{213}=0$ \\
\hline & & 2 & $x_{223}=0$ \\
\hline & & 3 & $x_{233}=0$ \\
\hline & Lower bound $f^{-}$ & & $\$ 260,670,295$ \\
\hline
\end{tabular}

$x_{133}+x_{233} \geq 300$

$x_{i j k} \geq 0 \quad \forall i, j, k$.

Solving using MAPLE-12, $f^{-}=\$ 260,670,295$ at the values given in Table 6 .

Further, using the model (UIQPP) for upper bound, we obtain

$$
\begin{aligned}
f^{+}= & \min _{x}\left(1 8 2 5 \left(57 x_{111}+84.595 x_{211}+54.39 x_{121}+85.345 x_{221}+58 x_{131}+83.215 x_{231}\right.\right. \\
& +74.3 x_{112}+105.584 x_{212}+71.58 x_{122}+105.364 x_{222}+75.54 x_{132}+103.134 x_{232} \\
& +88,45 x_{113}+120.185 x_{213}+85.35 x_{123}+121.075 x_{223}+89.7 x_{133}+118.585 x_{233} \\
& -0.0121 x_{111}^{2}-0.01121 x_{211}^{2}-0.0105 x_{121}^{2}-0.01441 x_{221}^{2}-0.0130 x_{131}^{2} \\
& -0.01041 x_{231}^{2}-0.0139 x_{112}^{2}-0.01255 x_{212}^{2}-0.0116 x_{122}^{2}-0.01295 x_{222}^{2} \\
& -0.0142 x_{132}^{2}-0.01145 x_{232}^{2}-0.0146 x_{113}^{2}-0.01354 x_{213}^{2} \\
& \left.\left.-0.0127 x_{123}^{2}-0.01414 x_{223}^{2}-0.0158 x_{133}^{2}-0.01254 x_{233}^{2}\right)\right)
\end{aligned}
$$

subject to

$$
\begin{aligned}
& 1825\left(x_{111}+x_{121}+x_{131}+x_{112}+x_{122}+x_{132}+x_{113}+x_{123}+x_{133}\right. \\
& \left.\quad+0.3\left(x_{211}+x_{221}+x_{231}+x_{212}+x_{222}+x_{232}+x_{213}+x_{223}+x_{233}\right)\right) \\
& \leq 3.75 \times 10^{6} \\
& x_{211}+x_{221}+x_{231} \leq 500, \\
& x_{212}+x_{222}+x_{232} \leq 500, \\
& x_{213}+x_{223}+x_{233} \leq 500, \\
& x_{111}+x_{211} \geq 350
\end{aligned}
$$


Table 7 Optimal values of variables for upper bound $f^{+}$

\begin{tabular}{|c|c|c|c|}
\hline Time span $(k)$ & Facility type (i) & Locality $(j)$ & Optimum waste allocation (tonnes) \\
\hline \multirow[t]{6}{*}{1} & 1 & 1 & $x_{111}=0$ \\
\hline & & 2 & $x_{121}=250$ \\
\hline & & 3 & $x_{131}=200$ \\
\hline & 2 & 1 & $x_{211}=350$ \\
\hline & & 2 & $x_{221}=0$ \\
\hline & & 3 & $x_{231}=150$ \\
\hline \multirow[t]{6}{*}{2} & 1 & 1 & $x_{112}=0$ \\
\hline & & 2 & $x_{122}=165$ \\
\hline & & 3 & $x_{132}=350$ \\
\hline & 2 & 1 & $x_{212}=400$ \\
\hline & & 2 & $x_{222}=100$ \\
\hline & & 3 & $x_{232}=0$ \\
\hline \multirow[t]{7}{*}{3} & 1 & 1 & $x_{113}=0$ \\
\hline & & 2 & $x_{123}=285$ \\
\hline & & 3 & $x_{133}=361$ \\
\hline & 2 & 1 & $x_{213}=440$ \\
\hline & & 2 & $x_{223}=0$ \\
\hline & & 3 & $x_{233}=39$ \\
\hline & Upper bound $f^{+}$ & & $\$ 473,418,686$ \\
\hline
\end{tabular}

$$
\begin{aligned}
& x_{121}+x_{221} \geq 250, \\
& x_{131}+x_{231} \geq 350, \\
& x_{112}+x_{212} \geq 400, \\
& x_{122}+x_{222} \geq 265, \\
& x_{132}+x_{232} \geq 350, \\
& x_{113}+x_{213} \geq 440, \\
& x_{123}+x_{223} \geq 285, \\
& x_{133}+x_{233} \geq 400, \\
& x_{i j k} \geq 0 \quad \forall i, j, k .
\end{aligned}
$$

It yields $f^{+}=\$ 473,418,686$ (using MAPLE-12). The values at which the function attains the upper limit are given in Table 7. The graphical interpretation of the results can be seen in Figs. 7-8. Here, LF stands for the landfill and IC stands for the incinerator.

Remark 2 The objective functions of the given WM problem dealing with $f^{-}$and $f^{+}$are of minimization type with Hessian matrices as diagonal of order $18 \times 18$ having all negative entries. This shows that the problems are having concave type objective functions subject to linear equations. Further, if we apply the duality principle for the concave minimization type objective function subject to linear constraints, the optimal values of the two problems may not be the same. For instance, consider the following example:

$$
\operatorname{Min} f(x)=-x^{2}-y^{2}+x+y
$$

subject to $0 \leq x \leq 1,0 \leq y \leq 1$.

It is to be noticed that the objective function in (E1) is concave subject to linear inequalities. Directly solving the problem, we get $x=1, y=1$, and $f(x)=0$ as the optimal solution. 


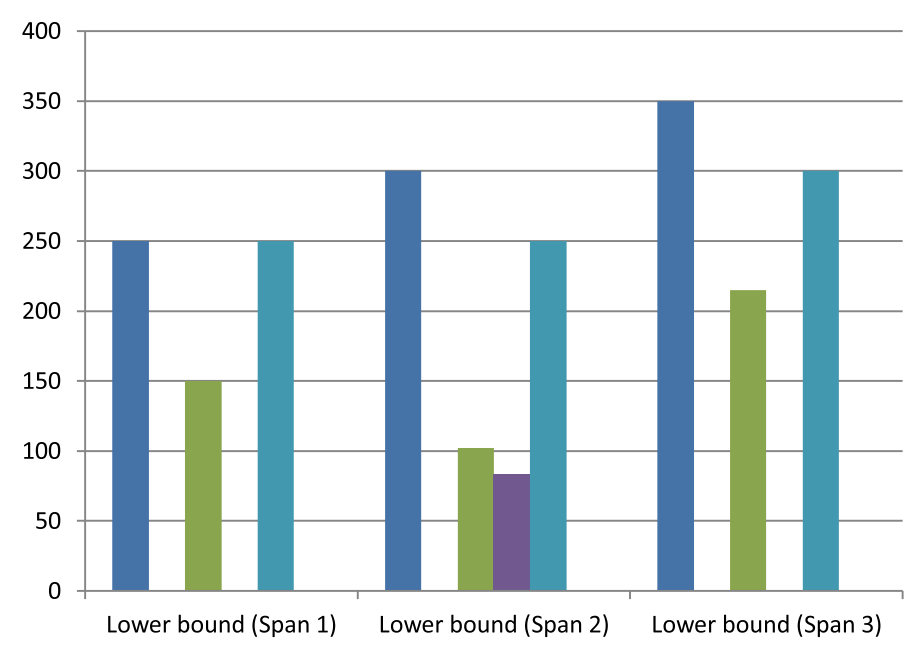

- Locality 1-LF

- Locality 1-IC

- Locality 2-LF

Locality 2 -IC

Locality 3-LF

Locality 3 -IC

Figure 7 Amount of waste allocation to achieve $f^{-}$

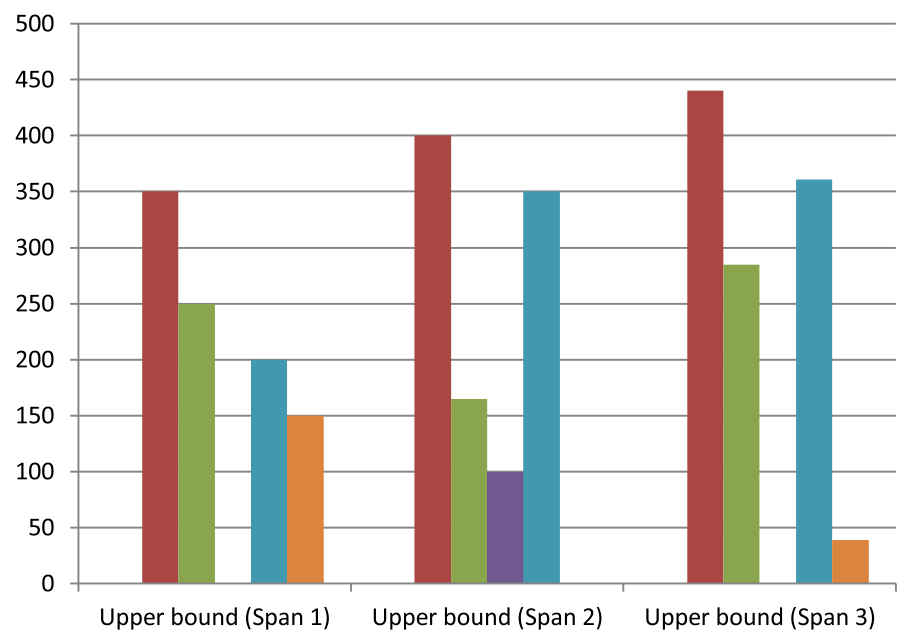

- Locality 1-LF

- Locality 1-IC

Locality 2-LF

- Locality 2 -IC

- Locality 3-LF

Locality 3 -IC

Figure 8 Amount of waste allocation to achieve $f^{+}$

However, on applying duality, we obtain

$\operatorname{Max} f(x)=x^{2}+y^{2}-\lambda_{1}-\lambda_{2}$

subject to $-2 x+\lambda_{1}-\delta_{1}=-1$,

$$
\begin{aligned}
& -2 y+\lambda_{2}-\delta_{2}=-1, \\
& x \geq 0, y \geq 0, \lambda_{1} \geq 0, \lambda_{2} \geq 0, \delta_{1} \geq 0, \delta_{2} \geq 0,
\end{aligned}
$$

which after solving yields $x=0.5, y=0.5$, and $f(x)=0.5$. This implies that the optimal values of (E1) and (E2) are not the same. It shows that duality is not applicable in (E1) as it is not a convex optimization problem (see p. 232, Bazaraa et al. [38]). 
Based on the above discussion, it can be concluded that the implementation of the duality based algorithm by Kong et al. [36] to a WM problem governed by an inexact concave quadratic programming is erroneous. The present article not only develops a method to solve IQP with concave type objective function, but also removes a deficiency in the execution of the duality algorithm to the WM problem in Kong et al. [36].

Computational efficiency of the proposed algorithm versus existing methods

In the literature, a vast number of WM problems follow a linear model due to the assumption of constant costs along with imprecise parameters. However, the concave optimization models dealing with WM in [12, 35] are tackled by derivative algorithm. Proposed algorithm scores over the existing algorithms in the following sense:

- The derivative algorithm introduced by Chen and Huang [35] involves $2 n$ variables and $2(m+n)$ constraints, whereas the proposed algorithm uses only $n$ variables and $m+n$ constraints, thus leading to a more computationally efficient algorithm.

- The procedure for derivative algorithm in [12, 35] is more cumbersome for application purposes in comparison to proposed algorithm which requires to identify only sense of the objective function and signs of squared terms.

- The duality based algorithm proposed by Kong et al. [36] for convex type quadratic programming problems involves $2 n+m$ variables and $4 n+m$ constraints which are also significantly higher in comparison to $n$ variables and $m+n$ constraints in the proposed algorithm.

\section{Conclusions}

The problems of WM are commonly governed by either LP problems or concave quadratic programming problems, depending upon constant or decreasing linear cost functions, respectively. The present article provides a solution methodology to tackle concave quadratic programming problems having inexact parameters. It is easy to apply and more computationally efficient in comparison to the derivative algorithm used in Li and Huang [12], Chen and Huang [35], and duality approach in Kong et al. [36]. Further, the discrepancy in the implementation of the algorithm to the application in the WM problem by Kong et al. [36] is also removed. In future, it will be interesting to investigate the algorithm for general model of quadratic/ nonlinear programming problem with inexact parameters and decision variables. Further, parabolic cost functions may be considered while dealing with application in WM.

\footnotetext{
Acknowledgements

The authors sincerely acknowledge the valuable suggestions and recommendations of the anonymous reviewers which have considerably improved the presentation and quality of the paper. The first author is also thankful to the Quality Improvement Programme Centre of IIT Roorkee along with All India Council of Technical Education for their support to carry out this work. The third and fourth authors thank the King Fahd University of Petroleum and Minerals for the support under the project no. SB191005.

Funding

This research is supported by the King Fahd University of Petroleum and Minerals, Dhahran, Saudi Arabia, under the Small/Basic Research Grant No. SB191005.
}

Availability of data and materials

Not applicable.

Competing interests

The authors declare that they have no competing interests.

Authors' contributions

All authors jointly worked on the results, and they read and approved the final manuscript. 


\section{Author details}

${ }^{1}$ Department of Applied Sciences, Punjab Engineering College, Chandigarh, 160012, India. ${ }^{2}$ Department of Mathematics, Indian Institute of Technology Roorkee, Roorkee, 247667, India. ${ }^{3}$ Department of Mathematics \& Statistics, King Fahd University of Petroleum \& Minerals, Dhahran, 31261, Kingdom of Saudi Arabia.

\section{Publisher's Note}

Springer Nature remains neutral with regard to jurisdictional claims in published maps and institutional affiliations.

Received: 9 December 2020 Accepted: 17 March 2021 Published online: 31 March 2021

\section{References}

1. Chang, N.B., Lu, H.Y.: A new approach for long term planning of solid waste management systems using fuzzy global criterion. J. Environ. Sci. Health, Part A 32(4), 1025-1047 (1997)

2. Sakai, S.I., Hiraoka, M.: Municipal solid waste incinerator residue recycling by thermal processes. Waste Manag. 20(2-3), 249-258 (2000)

3. Cai, Y., Huang, G.H., Nie, X.H., Li, Y.P., Tan, Q.: Municipal solid waste management under uncertainty: a mixed interval parameter fuzzy-stochastic robust programming approach. Environ. Eng. Sci. 24(3), 338-352 (2007)

4. Fan, Y.R., Huang, G.H., Li, Y.P., Cao, M.F., Cheng, G.H.: A fuzzy linear programming approach for municipal solid-waste management under uncertainty. Eng. Optim. 41(12), 1081-1101 (2009)

5. Sun, Y., Huang, G.H., Li, Y.P.: ICQSWM: an inexact chance-constrained quadratic solid waste management model. Resour. Conserv. Recycl. 54(10), 641-657 (2010)

6. Tan, Q., Huang, G.H., Cai, Y.P.: Identification of optimal plans for municipal solid waste management in an environment of fuzziness and two-layer randomness. Stoch. Environ. Res. Risk Assess. 24(1), 147-164 (2010)

7. Lu, H.W., Huang, G.H., He, L., Zeng, G.M.: An inexact dynamic optimization model for municipal solid waste management in association with greenhouse gas emission control. J. Environ. Manag. 90(1), 396-409 (2009)

8. He, L., Huang, G.H., Lu, H.: Greenhouse gas emissions control in integrated municipal solid waste management through mixed integer bilevel decision-making. J. Hazard. Mater. 193, 112-119 (2011)

9. Astrup, T., Riber, C., Pedersen, A.J.: Incinerator performance: effects of changes in waste input and furnace operation on air emissions and residues. Waste Manag. Res. 29(10-suppl), S57-S68 (2011)

10. Li, P., Chen, B.: FSILP: fuzzy-stochastic-interval linear programming for supporting municipal solid waste management. J. Environ. Manag. 92(4), 1198-1209 (2011)

11. Fan, Y.R., Huang, G.H.: A robust two-step method for solving interval linear programming problems within an environmental management context. J. Environ. Inform. 19(1), 1-9 (2012)

12. Li, Y., Huang, G.: Robust interval quadratic programming and its application to waste management under uncertainty. Environ. Syst. Res. 1(1), 7 (2012)

13. Ionescu, G., Rada, E.C., Ragazzi, M., Mărculescu, C., Badea, A., Apostol, T.: Integrated municipal solid waste scenario model using advanced pretreatment and waste to energy processes. Energy Convers. Manag. 76, 1083-1092 (2013)

14. Rada, E.C., Ragazzi, M., lonescu, G., Merler, G., Moedinger, F., Raboni, M., Torretta, V.: Municipal solid waste treatment by integrated solutions: energy and environmental balances. Energy Proc. 50, 1037-1044 (2014)

15. Xu, Y., Huang, G.H., Cheng, G.H., Liu, Y., Li, Y.F.: A two-stage fuzzy chance-constrained model for solid waste allocation planning. J. Environ. Inform. 24(2), 101-110 (2014)

16. Sugimoto, T., Fukushima, M., Ibaraki, T.: A parallel relaxation method for quadratic programming problems with interval constraints. J. Comput. Appl. Math. 60(1-2), 219-236 (1995)

17. Van Thoai, N.: Global optimization techniques for solving the general quadratic integer programming problem. Comput. Optim. Appl. 10(2), 149-163 (1998)

18. Gao, Y., Xue, H., Shen, P.: A new rectangle branch-and-reduce approach for solving nonconvex quadratic programming problems. Appl. Math. Comput. 168(2), 1409-1418 (2005)

19. Liu, S.T., Wang, R.T.: A numerical solution method to interval quadratic programming. Appl. Math. Comput. 189(2), 1274-1281 (2007)

20. Fan, Y.R., Huang, G.H., Li, Y.P.: Robust interval linear programming for environmental decision making unde uncertainty. Eng. Optim. 44(11), 1321-1336 (2012)

21. Fan, Y.R., Huang, G.H., Yang, A.L.: Generalized fuzzy linear programming for decision making under uncertainty: feasibility of fuzzy solutions and solving approach. Inf. Sci. 241, 12-27 (2013)

22. Atalay, K.D., Apaydin, A.: Gamma distribution approach in chance-constrained stochastic programming model J. Inequal. Appl. 2011, 108 (2011). https://doi.org/10.1186/1029-242X-2011-108

23. Zhu, H., Ni, Q., Zhang, X.: A simple approximated solution method for solving fractional trust region subproblems of nonlinearly equality constrained optimization. J. Inequal. Appl. 2020, 25 (2020). https://doi.org/10.1186/s13660-020-2300-7

24. Van Hung, N., Tam, V.M., Koebis, E., Yao, J.C.: Existence of solutions and algorithm for generalized vector quasi-complementarity problems with application to traffic network problems. J. Nonlinear Convex Anal. 20(9), $1751-1775$ (2019)

25. Van Hung, N., Tam, V.M., Tuan, N.H., O'Regan, D.: Convergence analysis of solution sets for fuzzy optimization problems. J. Comput. Appl. Math. 369, 112615 (2020)

26. Van Hung, N., Tam, V.M., O'Regan, D., Cho, Y.J.: A new class of generalized multiobjective games in bounded rationality with fuzzy mappings: structural $(\lambda, \epsilon)$-stability and $(\lambda, \epsilon)$-robustness to $\epsilon$-equilibria. J. Comput. Appl. Math. $372,112735(2020)$

27. Van Hung, N., Keller, A.A.: Painlevé-Kuratowski convergence of the solution sets for controlled systems of fuzzy vector quasi-optimization problems with application to controlling traffic networks under uncertainty. Comput. Appl. Math. 40(1), 1-21 (2021)

28. Li, Y.P., Huang, G.H., Nie, S.L.: Mixed interval-fuzzy two-stage integer programming and its application to flood-diversion planning. Eng. Optim. 39, 163-183 (2007) 
29. Li, Y.P., Huang, G.H.: Inexact multistage stochastic quadratic programming method for planning water resources systems under uncertainty. Environ. Eng. Sci. 24(10), 1361-1378 (2007)

30. Fan, Y., Huang, G., Veawab, A.: A generalized fuzzy linear programming approach for environmental management problem under uncertainty. J. Air Waste Manage. Assoc. 62(1), 72-86 (2012)

31. Jin, L., Huang, G., Fan, Y., Nie, X., Cheng, G.: A hybrid dynamic dual interval programming for irrigation water allocation under uncertainty. Water Resour. Manag. 26, 1183-1200 (2012)

32. Miao, D.Y., Huang, W.W., Li, Y.P., Yang, Z.F.: Planning water resources systems under uncertainty using an interval-fuzzy de novo programming method. J. Environ. Inform. 24(1), 11-23 (2014)

33. Zhang, N., Li, Y.P., Huang, W.W., Liu, J.: An inexact two-stage water quality management model for supporting sustainable development in a rural system. J. Environ. Inform. 24(1), 52-64 (2014)

34. Huang, W., Dai, L., Baetz, B., Cao, M., Razavi, S.: Interval binary programming model for noise control within an urban environment. J. Environ. Inform. 21, 93-101 (2015)

35. Chen, M.J., Huang, G.H.: A derivative algorithm for inexact quadratic program-application to environmental decision-making under uncertainty. Eur. J. Oper. Res. 128(3), 570-586 (2001)

36. Kong, X.M., Huang, G.H., Fan, Y.R., Li, Y.P.: A duality theorem-based algorithm for inexact quadratic programming problems: application to waste management under uncertainty. Eng. Optim. 48(4), 562-581 (2016)

37. Hoffman, K.L.: A method for globally minimizing concave functions over convex sets. Math. Program. 20(1), 22-32 (1981)

38. Bazaraa, M.S., Sherali, H.D., Shetty, C.M.: Nonlinear Programming: Theory and Algorithms. Wiley, New York (2004)

\section{Submit your manuscript to a SpringerOpen ${ }^{\circ}$ journal and benefit from:}

- Convenient online submission

- Rigorous peer review

- Open access: articles freely available online

- High visibility within the field

- Retaining the copyright to your article

Submit your next manuscript at $\mathbf{s p r i n g e r o p e n . c o m ~}$ 